\title{
Antibody ligation of CM1 on cisplatin-exposed HeLa cells induces apoptosis through reactive oxygen species-dependent Fas ligand expression
}

\author{
GA BIN PARK $^{1 *}$, DAEJIN KIM ${ }^{1 *}$, HOI SOO YOON ${ }^{1}$, YEONG-SEOK KIM $^{1}$, HYUN-KYUNG LEE $^{2}$, \\ KI TAE KIM ${ }^{3}$, DAE HOON JEONG ${ }^{3}$ and DAE YOUNG HUR ${ }^{3}$ \\ ${ }^{1}$ Department of Anatomy and Research Center for Woman Disease, Inje University College of Medicine; \\ Departments of ${ }^{2}$ Internal Medicine and ${ }^{3}$ Obstetrics and Gynecology, Inje University \\ Busan Paik Hospital, Busan 614-735, Republic of Korea
}

Received December 19, 2013; Accepted February 27, 2014

DOI: 10.3892/ijo.2014.2361

\begin{abstract}
Centrocyte/centroblast marker 1 (CM1) has been identified as a pro-apoptosis molecule on B-cell lymphoma cells as well as several types of cancer cells. In this study, we investigated its signaling mechanism in HeLa cells after treatment with cisplatin in order to potentially identify a new therapeutic target. The CM1 molecule was induced on the surface of cisplatin-exposed HeLa cells. In these cells, ligation of CM1 with anti-CM1 monoclonal antibodies inhibited cell proliferation and produced reactive oxygen species. Fas ligand (FasL) expression was upregulated without upregulating Fas in cisplatin-exposed HeLa cells after CM1 stimulation. Pretreatment with $\mathrm{N}$-acetylcysteine, a pan-capase inhibitor, and ZB4, an antagonistic anti-Fas antibody, effectively inhibited the apoptotic effect triggered by CM1. CM1 ligation induced apoptosis through disruption of the mitochondrial membrane potential, decreased Bcl-2 and phosphorylated ERK expression. These findings identify CM1 as a potential new therapeutic target related to cisplatin-exposed cervical cancer.
\end{abstract}

\section{Introduction}

Human papillomavirus (HPV)-associated cervical cancer is the third most commonly diagnosed cancer and the fourth

Correspondence to: Professor Dae Young Hur, Department of Anatomy, Inje University, College of Medicine, Bokji-ro 75, Busanjin-Gu, Busan 614-735, Republic of Korea

E-mail: dyhur@inje.ac.kr

Professor Dae Hoon Jeong, Department of Obstetrics and Gynecology, Inje University Busan Paik Hospital, Busan 614-735, Republic of Korea

E-mail: dhjeong@inje.ac.kr

${ }^{*}$ Contributed equally

Key words: cisplatin, HeLa cells, ROS, Fas ligand, CM1 leading cause of cancer deaths in women worldwide (1). While the broad use of Papanicolaou (Pap) screening and conventional treatment have led to a decline in mortality from cervical cancer, many women still die of the disease in developing countries (2). When detected at an early stage, invasive cervical cancer is one of the most successfully treated cancers. However, the 5-year relative survival rate drops to $13-46 \%$ if detected at an advanced stage (3). Invasive cervical cancer is generally treated with a combination of surgery, radiation, and/ or chemotherapy. Cisplatin-based chemotherapy has traditionally been reserved for metastatic or recurrent cervical cancer (4).

Cisplatin, a DNA-damaging cancer treatment that readily induces apoptosis in vitro, is widely used in a variety of human carcinomas, including head and neck, colorectal, ovarian, cervical, testicular, and small cell lung cancer (5). A major limitation of cisplatin-based chemotherapy is the development of cellular resistance, which is implicated in its failure to treat malignant tumors $(6,7)$. The mechanisms of cisplatin resistance have been identified as a reduction in drug uptake, the induction of DNA repair enzymes such as Bcl-2, the enhancement of anti-oxidant activity with glutathione, and the inhibition of caspase activity (7-9). Based on the accumulated evidence, various experimental and clinical trials are under investigation to overcome these mechanisms of drug resistance. New cisplatin-based combination therapies are necessary to improve outcomes in advanced cervical cancer.

CM1 has been identified as a pro-apoptosis molecule on B-cell lymphoma cells. It was first identified by a monoclonal antibody developed against concanavalin-A stimulated mononuclear cells in the peripheral blood. CM1 molecules are distributed across germinal centers in the human tonsil and expressed on activated T and B lymphocytes (10). It has previously been reported that cross-linking CM1 with anti-CM1 antibody induces apoptosis in Burkitt's lymphoma cells, EBV-transformed B cells, and lung cancer cells. These effects are primarily mediated by activation of the caspase cascade and generation of reactive oxygen species (ROS) (11-13). These observations encouraged us to evaluate whether HeLa cervical cancer cells express CM1, which we found to be the 
case after exposure to cisplatin. Therefore, this study aimed to investigate the detailed signal mechanism of CM1-mediated apoptosis in cisplatin-exposed HeLa cells.

\section{Materials and methods}

Cells and reagents. Cells from the HeLa cervical cancer cell line were obtained from the American Type Culture Collection (ATCC, Manassas, VA, USA). Cells were grown and maintained in RPMI-1640 medium (Hyclone, Logan, UT, USA) containing $2 \mathrm{mM}$ L-gultamine, $100 \mathrm{U} / \mathrm{ml}$ penicillin, $100 \mu \mathrm{g} / \mathrm{ml}$ streptomycin and $10 \%$ heat-inactivated fetal bovine serum (Hyclone) at $37^{\circ} \mathrm{C}$ in a $5 \% \mathrm{CO}_{2}$ incubator. Anti-CM1 monoclonal antibody was generated using a murine hybridoma cell line-secreting antibody, as previously described (10). Cisplatin, N-acetylcysteine (NAC), and phorbol myristate acetate (PMA) were purchased from Sigma-Aldrich (St. Louis, MO, USA); 3, 3'-dihexyloxacarbocyanine iodide ( DiOC $_{6}$ ) and 2',7'-dichlorodihydrofluorescein diacetate (DCFH-DA) were purchased from Molecular Probes (Eugene, OR, USA). Fluorescein isothiocyanate (FITC)-conjugated anti-Fas and anti-Fas ligand monoclonal antibodies were purchased from BD Bioscience (San Jose, CA, USA). Rabbit anti-mouse IgG antibody, secondary antibody, and MOPC21 isotype control antibody were purchased from Sigma-Aldrich. Mouse antihuman apoptosis inducing factor (AIF) antibody and goat anti-human endonuclease $\mathrm{G}$ (EndoG) antibody were purchased from Santa Cruz Biotechnologies (Santa Cruz, CA, USA). Z-VAD-fmk, a pan-caspase inhibitor, was purchased from Calbiochem (La Jolla, CA, USA).

Cell proliferation assay. HeLa cells were pre-incubated with each concentration of cisplatin (0-100 $\mu \mathrm{M})$ for $48 \mathrm{~h}$, then cultured in media containing $10 \%$ FBS in a 96 -well flat-bottom plate $\left(5 \times 10^{4}\right.$ cells/well). Cell proliferation was estimated using an AlamarBlue assay kit (Serotec, Raleigh, NC, USA) as previously described (13). To analyze the effect of CM1 ligation on cell proliferation, cisplatin-exposed HeLa cells were cross-linked with immobilized anti-CM1 antibody $\left(\mathrm{IgG}_{1 \kappa}, 10 \mu \mathrm{g} / \mathrm{ml}\right)$ and MOPC21 ( $\mathrm{IgG}_{1 \kappa}$; Sigma-Aldrich) as isotype control for $40 \mathrm{~min}$. Next, cells were cultured in media containing $10 \% \mathrm{FBS}$ at $37^{\circ} \mathrm{C}$ for $48 \mathrm{~h}$ in a 96-well flat-bottom plate $\left(2 \times 10^{5}\right.$ cells/well). To immobilize the anti-CM1 and MOPC21 antibodies, $10 \mu \mathrm{g} / \mathrm{ml}$ of each antibody in phosphatebuffered saline (PBS) were applied to a 96-well culture plate $(0.1 \mathrm{ml} /$ well; wells were washed with PBS before use $)$ after overnight incubation at $4^{\circ} \mathrm{C}$. Cell proliferation was estimated using an AlamarBlue assay kit (Serotec). Briefly, AlamarBlue was added ( $10 \%$ by total volume) to each well, and the relative fluorescence value was tested $7 \mathrm{~h}$ later using a fluorometer (Synergy HT, Bio-Tek Instruments Inc., Winnoski, VT; excitation, $530 \mathrm{~nm}$; emission, $590 \mathrm{~nm}$ ).

Analysis of CM1 expression with flow cytometry and confocal microscopy. Flow cytometry was employed to evaluate the expression of surface CM1 molecules. First, HeLa cells were incubated with or without cisplatin at various concentrations $(0,10,20,50$ and $100 \mu \mathrm{M})$. Cells were washed twice with ice-cold PBS, then incubated with FITC-conjugated mouse anti-human CM1 antibodies (mouse $\mathrm{IgG}_{1}$ ) for $30 \mathrm{~min}$ on ice, followed by 2 more washes with ice-cold PBS. To detect intracellular molecules, HeLa cells were treated with a permeabilization buffer $(0.1 \%$ saponin in PBS) before staining with FITC-conjugated mouse anti-human CM1 antibodies. All samples were assessed with a FACSCalibur flow cytometer (BD Bioscience) and data were processed by the program CellQuest (BD Bioscience). To confirm CM1 expression with confocal microscopy, HeLa cells were pre-incubated with $20 \mu \mathrm{M}$ cisplatin, with $20 \mathrm{ng} / \mathrm{ml}$ PMA, or with nothing for $48 \mathrm{~h}$. They were then incubated with FITC-conjugated anti-CM1 antibody. Fluorescence-stained cells were examined by confocal laser-scanning microscopy (510 META, Carl Zeiss, Jena, Germany) at x400 magnification, and images were acquired with Confocal Microscopy Software Release 3.0 (510 META, Carl Zeiss).

Detection of CM1-mediated apoptosis. For immobilization, anti-CM1 and MOPC21 antibodies were incubated overnight at $4^{\circ} \mathrm{C}$ on a 96 -well culture plate $(10 \mu \mathrm{g} / \mathrm{ml}, 250 \mu \mathrm{l}, 2.5 \mu \mathrm{g} / \mathrm{well})$. To evaluate apoptosis by CM1 cross-linking, HeLa cells were treated with cisplatin $(20 \mu \mathrm{M})$ for $48 \mathrm{~h}$ and then $5 \times 10^{5}$ cells/ well were cross-linked with immobilized anti-CM1 antibody or MOPC21 isotype control antibody (2.5 $\mu \mathrm{g} /$ well). Next, cells were incubated at $37^{\circ} \mathrm{C}$ for the indicated amount of time. Following treatment, cells were washed twice with cold PBS then analyzed for Annexin V expression by flow cytometry as previously described (13).

Measurement of mitochondrial membrane potential and ROS generation. To measure ROS levels and mitochondrial membrane potentials $(\Delta \psi)$, cisplatin-exposed HeLa cells were cultured with DCFH-DA at $37^{\circ} \mathrm{C}$ for 30 min or $\mathrm{DiOC}_{6}$ at $37^{\circ} \mathrm{C}$ for $15 \mathrm{~min}$ as molecular probes. Cells were further incubated with anti-CM1 antibody as described above. Cells were harvested at the indicated time and their ROS levels and $\Delta \psi$ were determined by an FACSCalibur flow cytometer (BD Bioscience).

Apoptosis-blocking experiments. To investigate the effects of caspase inhibitors and ROS on CM1-mediated apoptosis, cisplatin-exposed HeLa cells as described above were pre-treated with z-VAD-fmk (20 $\mu \mathrm{M}$ in DMSO, a broad-spectrum caspase inhibitor) or NAC for $2 \mathrm{~h}$ before antibody stimulation. Cells were further incubated with anti-CM1 antibody as described above. The apoptosis-blocking effects of z-VAD-fmk and NAC were detected using Annexin V, DCFH-DA and DiOC $_{6}$ staining as described above. To block Fas-FasL interaction, antagonistic anti-Fas antibody ZB4 $(0.5 \mathrm{mg} / \mathrm{ml})$ was added $2 \mathrm{~h}$ before treatment with anti-CM1 antibody. ZB4 was removed from cell cultures before stimulation with anti-CM1 antibody. Apoptosis was determined by flow cytometry after staining with Annexin V.

Confocal microscopy to detect apoptosis-related intracellular molecules. To detect intracellular apoptosis-related molecules, cisplatin-exposed HeLa cells were incubated with anti-CM1 antibody as described above. To detect the blocking effects of z-VAD-fmk nd NAC, cells were pre-treated with each substance for $2 \mathrm{~h}$ before antibody stimulation. Cells were incubated with primary antibodies against cytochrome $c$ (mouse $\mathrm{IgG} 2 \mathrm{~b}$ ) or AIF (mouse IgG2b) and then incubated with FITC-conjugated 
A

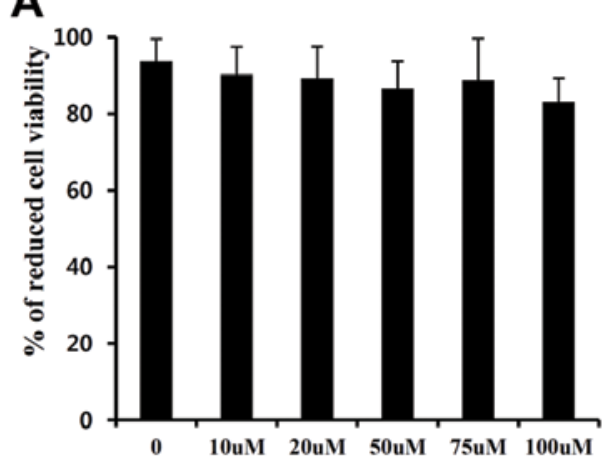

C

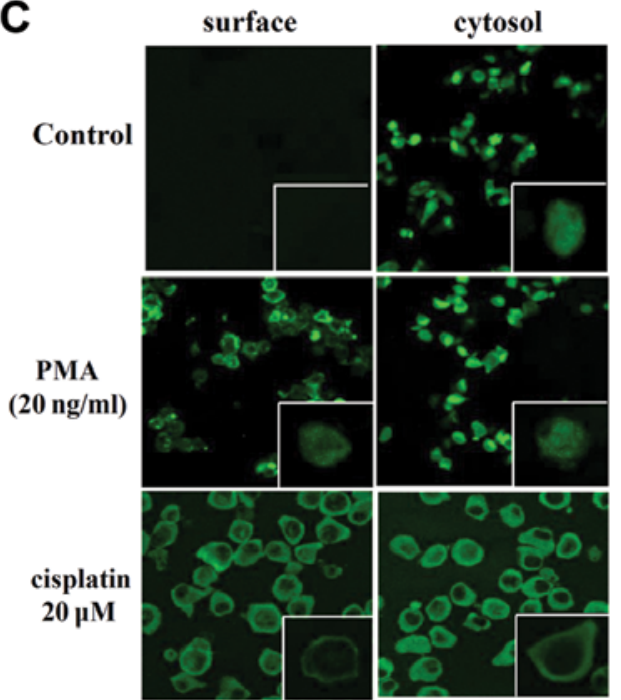

B

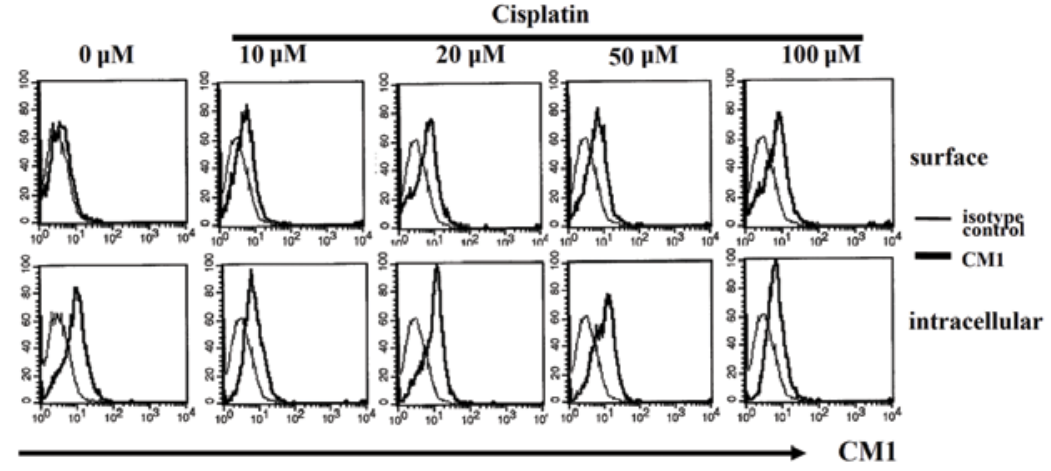

D

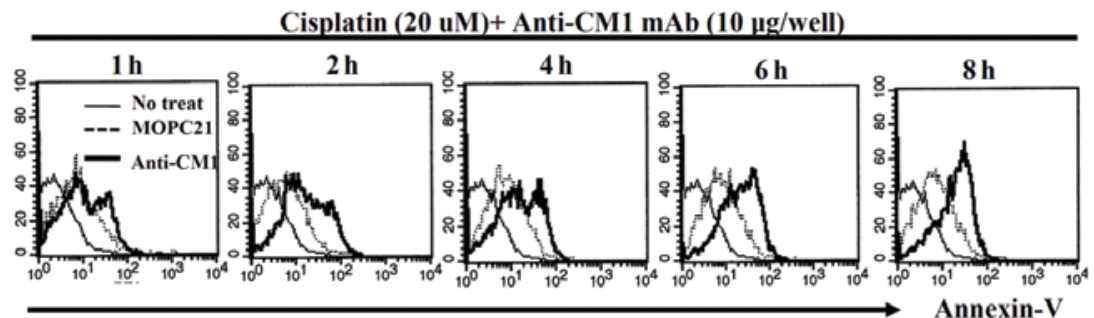

Figure 1. Effect of CM1 expression on HeLa cell proliferation. (A) Proliferation or viability of HeLa cells was determined by Alamar blue assay after culturing for $48 \mathrm{~h}$ in the presence of increasing concentrations of cisplatin. (B) Flow cytometry evaluation of CM1 expression on HeLa cells with or without cisplatin treatment (thin line, isotype control; thick line, FITC conjugated anti-CM1). (C) Confocal microscopy of CM1 expression on HeLa cells after $20 \mathrm{ng} / \mathrm{ml}$ PMA or $20 \mu \mathrm{M}$ cisplatin treatment. (D) Cisplatin-exposed HeLa cells were incubated (5x10 cells/well, $200 \mu 1)$ in an anti-CM1 or MOPC antibody-coated $(10 \mu \mathrm{g} / \mathrm{ml}, 250 \mu \mathrm{l}, 2.5 \mu \mathrm{g} / \mathrm{well})$ plate. Cells were harvested at the indicated time points. FITC-tagged Annexin V staining was performed to assess apoptosis. Dashed line histograms represent staining with isotype-matched antibody. Bold line histograms represent staining with FITC-tagged Annexin V. Each experiment is representative of three independent experiments.

goat anti-mouse $\operatorname{IgG}$ for 30 min. Nuclei were stained with PI for $10 \mathrm{~min}$ at room temperature. After being washed 3 times with PBS, the fluorescence-stained cells were examined under a confocal laser-scanning microscope (Carl Zeiss, 510 META) at $\mathrm{x} 400$ original magnification using Confocal Microscopy Software Release 3.0 (Carl Zeiss, 510 META).

Reverse-transcription polymerase chain reaction. Total RNA was isolated using an RNeasy Mini kit (Qiagen, Hilden, Germany). RNA was transcribed into cDNA using oligo (dT) primers (Bioneer, Daejeon, Korea) and reverse transcriptase. Polymerase chain reaction (PCR) amplification was performed using specific primer sets (Bioneer) for the Fas ligand (upstream primer, 5'-GGT CCA TGC CTC TGG AAT GG; downstream primer, 5'-CAC ATC TGC CCA GTA GTG CA, 250-bp product), Bcl-2 (upstream primer, 5'-GGA TTG TGG CCT TCT TTG AG; downstream primer, 5'-CAG CCA GGA GAA ATC AAA CAT, 209-bp product), and Bad (upstream primer, 5'-CGA GTG AGC AGG AAG ACT CC; downstream primer, 5'-CTG TGC TGC CCA GAG GTT, 299-bp product). For the control group, a specific primer set for $\beta$-actin was used (upstream primer, 5'-ATC CAC GAA ACT ACC TTC AA; downstream primer, 5'-ATC CAC ACG GAG TAC TTG C), which yielded a 200 -bp product. PCR (25 cycles; $20 \mathrm{sec}$ at $94^{\circ} \mathrm{C}, 10 \mathrm{sec}$ at $60^{\circ} \mathrm{C}$, and $30 \mathrm{sec}$ at $72^{\circ} \mathrm{C}$ ) was performed using Prime Taq Premix (GeNet Bio, Chungnam, Korea). The PCR products were visualized on $2.5 \%$ agarose gels with ethidium bromide.

Western blot analysis for apoptosis-related proteins. Cisplatinexposed HeLa cells were incubated with anti-CM1 antibody $(10 \mu \mathrm{g} / \mathrm{ml})$ as described above. Cells were harvested and washed twice with PBS. Cells were lysed in RIPA buffer (Elpis Biotech, Daejeon, Korea) containing a protease inhibitor cocktail (AEBSF, aprotinin, bestatin hydrochloride, E-64, EDTA, and leupeptin hemisulfate salt; Sigma-Aldrich). For western blot analysis, an equal amount of protein $(40 \mu \mathrm{g})$ was separated by electrophoresis on SDS-polyacrylamide gels and transferred to polyvinylidene difluoride membranes (Amersham Biosciences, Braunschweig, Germany) by electroblotting. Subsequently, membranes were incubated overnight at $4^{\circ} \mathrm{C}$ in a solution of PBS supplemented with 5\% non-fat dry milk. Blots 
A

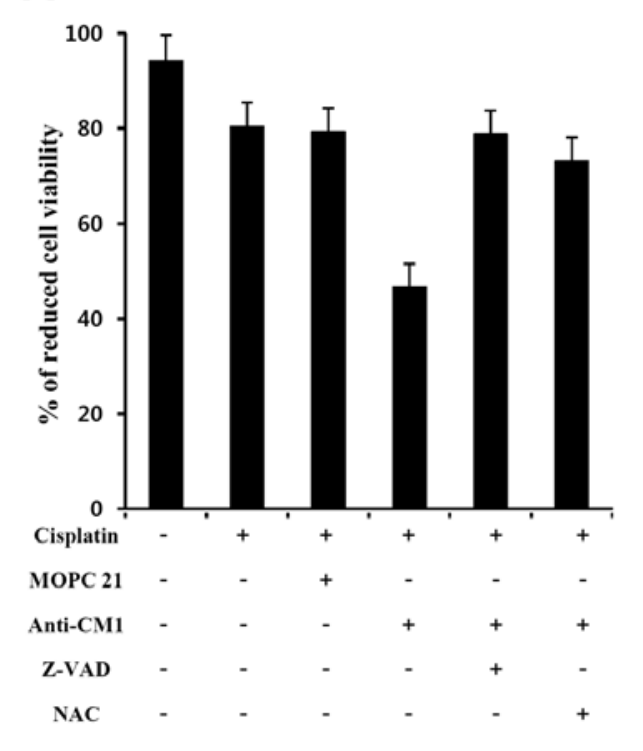

B
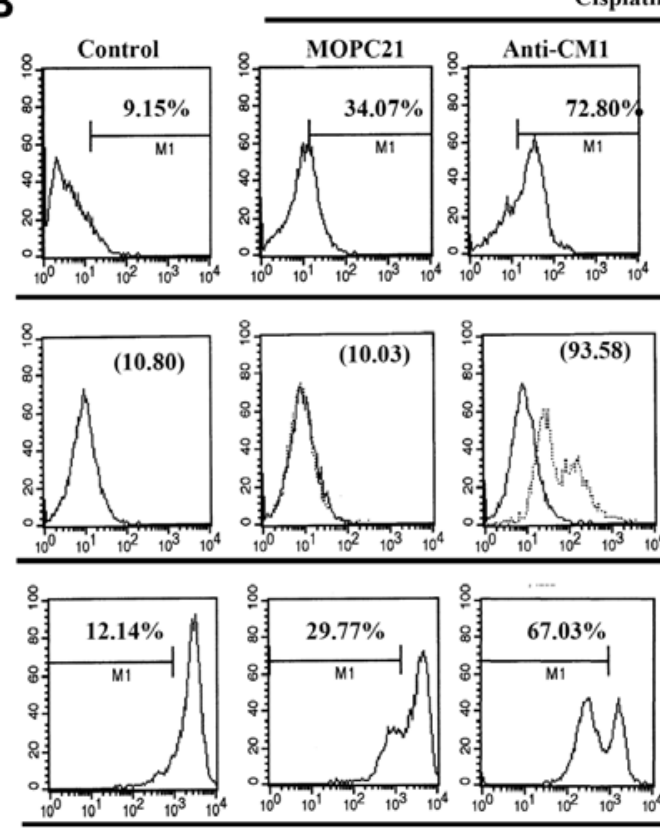

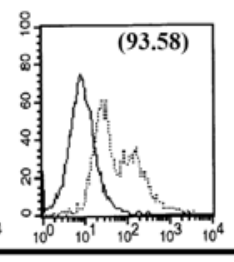

Cisplatin $(20 \mu \mathrm{M})$

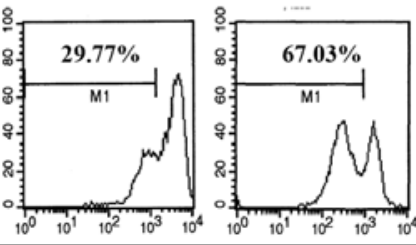

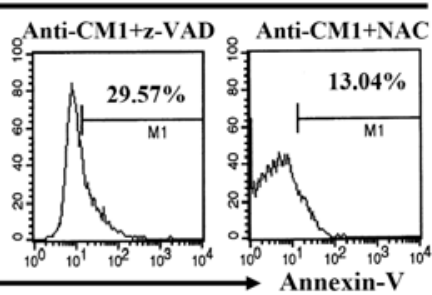
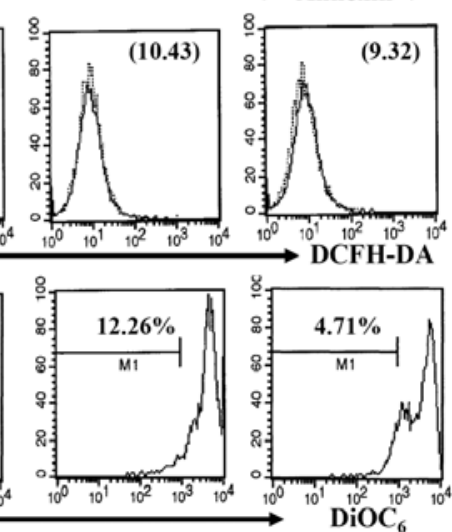

Figure 2. Effects of pan-caspase inhibitor z-VAD-fmk (z-VAD) and ROS quencher NAC on CM1-mediated apoptosis of cisplatin-exposed HeLa cells. (A) Measurement of proliferation in cisplatin-exposed HeLa cells after CM1 cross-linking. Cell proliferation was determined by Alamar Blue assay as described in Materials and methods. Data are representative of 5 independent experiments. (B) Cisplatin (20 $\mu \mathrm{M})$-exposed HeLa cells (5x10 ${ }^{5}$ cells/well, $\left.200 \mu \mathrm{l}\right)$ were pre-incubated with various inhibitors (z-VAD, NAC). The cells were washed with PBS and further incubated (5x10 5 cells/well, $200 \mu 1)$ in anti-CM1 antibody-coated $(2.5 \mu \mathrm{g} /$ well $)$ plates for $40 \mathrm{~min}$. Cells were then cultured in media containing $10 \%$ FBS at $37^{\circ} \mathrm{C}$ overnight. FITC-conjugated Annexin $\mathrm{V}$ staining was performed to assess apoptosis after overnight incubation. The number of DiOC ${ }_{6}$ represents the percentage of disrupted mitochondrial membrane potentials. To measure ROS generation, cells were pre-incubated with DCFH-DA before treatment with anti-CM1 or MOPC antibodies. Thin-line and dotted histograms represent ROS level of isotype control and anti-CM1 antibody-treated cells, respectively. The number in DCF histograms indicates the MFI. Results are representative of three independent experiments.

were probed with the desired antibodies [caspase-8, caspase-3, $\beta$-actin, Bid, Bcl-2, phospho-ERK, ERK and poly-ADP-ribose polymerase (PARP)] for $1 \mathrm{~h}$, incubated with diluted enzymelinked secondary antibody, and then visualized by enhanced chemiluminescence as instructed by the manufacturer (Amersham Bioscience). Protein loading equivalence was assessed by the expression of $\beta$-actin. Each experiment was repeated at least in triplicate.

\section{Results}

Ligation of CM1 with anti-CM1 antibody causes apoptosis in cisplatin-exposed HeLa cells. Cell viability of HeLa cells treated with cisplatin was not significantly reduced compared to the control group based on Alamar blue assay (Fig. 1A). Although CM1 is present in the cytoplasm of HeLa cells, it is not present on the cell surface until after exposure to cisplatin (Fig. 1B), as evidenced by confocal microscopic data (Fig. 1C). To determine the function of the surface CM1 molecule induced by cisplatin, HeLa cells were cross-linked with immobilized anti-CM1 antibody $(10 \mu \mathrm{g} / \mathrm{ml})$ or MOPC21 isotype control antibody. Stimulation of surface CM1 with anti-CM1 antibody increased the quantity of Annexin V-positive apoptotic cells in a time-dependent manner (Fig. 1D). These results suggest that surface CM1 promotes apoptosis in HeLa cells.

Cross-linking of CM1 induces apoptosis by ROS generation and caspase activation in cisplatin-exposed HeLa cells. We examined whether CM1-mediated apoptosis was related to ROS production or caspase activity following cross-linking with anti-CM1 antibody. Cisplatin-exposed $(20 \mu \mathrm{M}) \mathrm{HeLa}$ cells were pre-incubated with Z-VAD-fmk, a pan-caspase inhibitor, or NAC, a ROS inhibitor for $2 \mathrm{~h}$ before stimulation with anti-CM1 antibody. Cross-linking CM1 with immobilized anti-CM1 antibody inhibited cell proliferation when compared to cells stimulated with isotype antibody. We found that both Z-VAD-fmk and NAC pretreatment reversed the inhibition caused by anti-CM1 antibody stimulation (Fig. 2A). Furthermore, Z-VAD-fmk and NAC pretreatment also blocked CM1-mediated apoptosis in cisplatin-exposed HeLa cells (Fig. 2B). These results suggest that CM1-mediated apoptosis is associated with ROS generation and caspase activity in cisplatin-exposed HeLa cells.

CM1 stimulation on cisplatin-exposed HeLa cells results in mitochondrial release of AIF and EndoG. After observing that CM1 ligation disrupted mitochondrial membrane potentials, we used confocal microscopy to measure changes in the expression of apoptosis-inducing factor (AIF) and EndoG, which are stored in the mitochondria. Cross-linking CM1 with anti-CM1 antibody resulted in a significant release of AIF and EndoG from the mitochondria into the cytoplasm. Again, treatment with the pan-caspase inhibitor z-VAD-fmk and the ROS quencher NAC prevented this translocation of both AIF and EndoG (Fig. 3). These results suggest that CM1-mediated apoptosis in cisplatinexposed HeLa cells involves the mitochondria. 
A

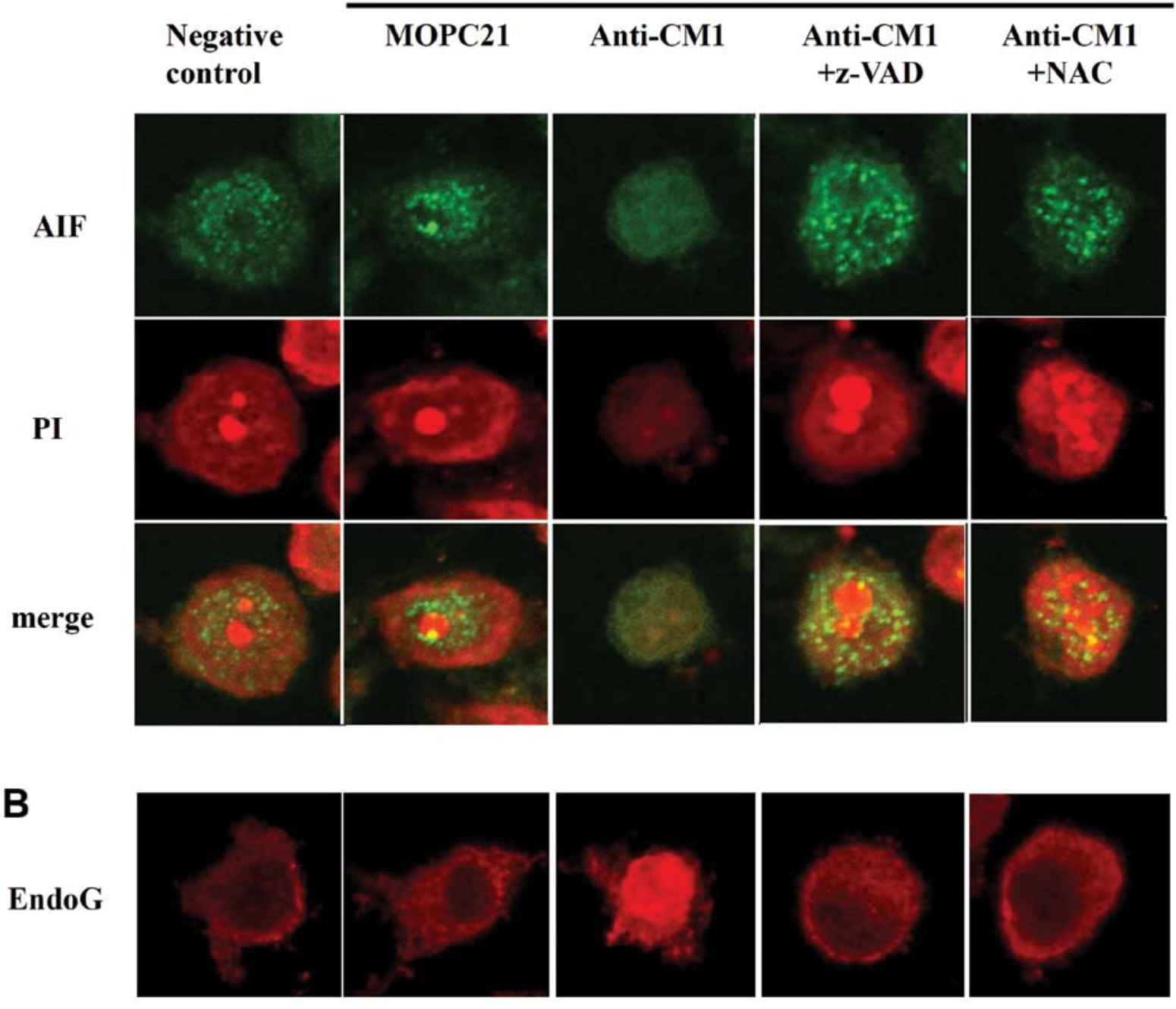

Figure 3. Subcellular distribution of AIF and EndoG after CM1 cross-linking in cisplatin-treated HeLa cells. Cisplatin (20 $\mu$ M)-exposed HeLa cells (5x10 cells/well, $200 \mu$ l) were pre-incubated with various inhibitors (z-VAD-fmk, NAC) before stimulation of CM1 with anti-CM1 antibody. Cells were harvested and permeabilized with $0.1 \%$ saponin in PBS. FITC conjugated anti-AIF (A) and PE-conjugated EndoG (B) antibodies were used. Nuclei were stained with PI (AIF, green fluorescence; EndoG and PI, red fluorescence). Cells were observed under confocal microscope (x400 magnification). These figures are representative of three independent experiments.

Cross-linking of CM1 induces Fas-mediated apoptosis in cisplatin-exposed HeLa cells. It is well-known that the death receptor and its ligand can initiate apoptosis. As described above, we observed that Z-VAD-fmk pretreatment prevents CM1-mediated apoptosis in cisplatin-exposed HeLa cells. In addition, Z-IETD-fmk, a caspase- 8 specific inhibitor, also blocks CM1-mediated apoptosis in cisplatin-exposed HeLa cells (Fig. 4A). We also investigated whether the Fas/ FasL interaction was involved in CM1-mediated apoptosis in cisplatin-exposed HeLa cells. HeLa cells expressed the Fas molecule constitutively, but did not express FasL. Although Fas expression was not affected by CM1 cross-linking, FasL expression was dramatically increased (Fig. 4B). We also foud that pre-treatment with $\mathrm{ZB} 4$, an antagonistic anti-Fas antibody, for $2 \mathrm{~h}$ blocked CM1-mediated apoptosis, although FasL expression was unchanged (Fig. 4C). These results suggest that CM1 ligation on cisplatin-exposed HeLa cells affects FasL expression, and that the Fas/FasL interaction is closely involved in CM1-mediated apoptosis.
CM1 ligation induces changes in apoptosis-related genes and activation of ERK and caspase in cisplatin-exposed HeL a cells. To investigate the detailed signaling mechanism of CM1-mediated apoptosis in cisplatin-exposed HeLa cells, we examined the expression of genes related to mitochondrial membrane disruption using RT-PCR. After CM1 ligation, FasL mRNA expression significantly increased, Bad (a proapoptotic gene) mRNA expression slightly increased, and Bcl-2 mRNA expression significantly decreased (Fig. 5A). Moreover, expression of Bcl-2 and Bid proteins, both antiapoptotic proteins, also decreased significantly after CM1 cross-linking (Fig. 5B). Both z-VAD-fmk and NAC inhibited these changes in mRNA and protein expression.

Next, we examined the activation of ERK1/2, a mitogen-activated protein kinase (MAPK), which regulates apoptosis-related genes. CM1 ligation induced the phosphorylation of ERK1/2 (Fig. 5B), as well as the activation of caspase- 8 and -3 , and the cleavage of PARP (Fig. 5C). Pretreatment with z-VAD-fmk, NAC, and ZB4 blocked these changes in apoptosis-related 
A

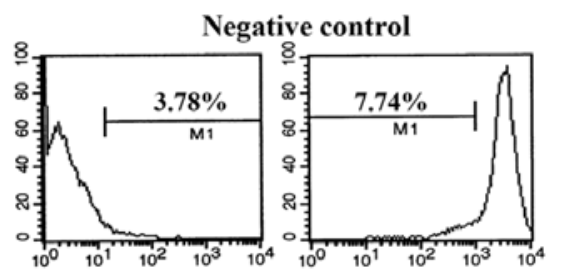

Cisplatin $(20 \mu \mathrm{M})+\mathrm{MOPC} 21$
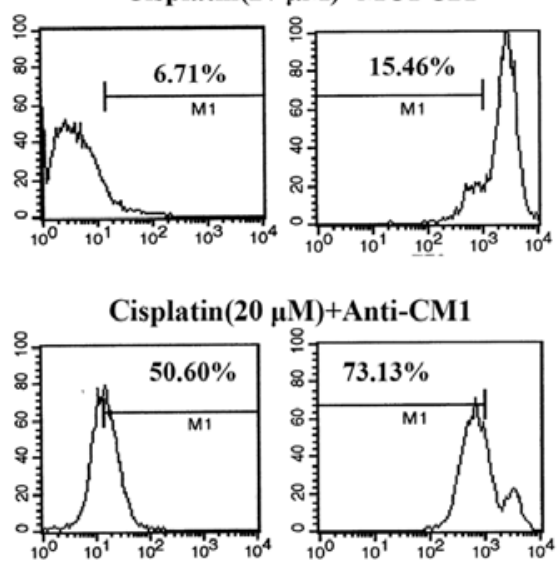

Cisplatin $(20 \mu \mathrm{M})+$ Anti-CM1+z-IETD

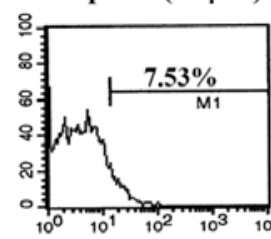

Annexin-V

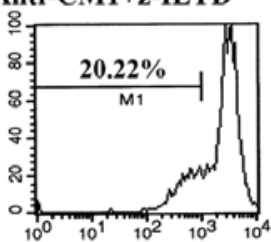

$\mathrm{DiOC}_{6}$
B

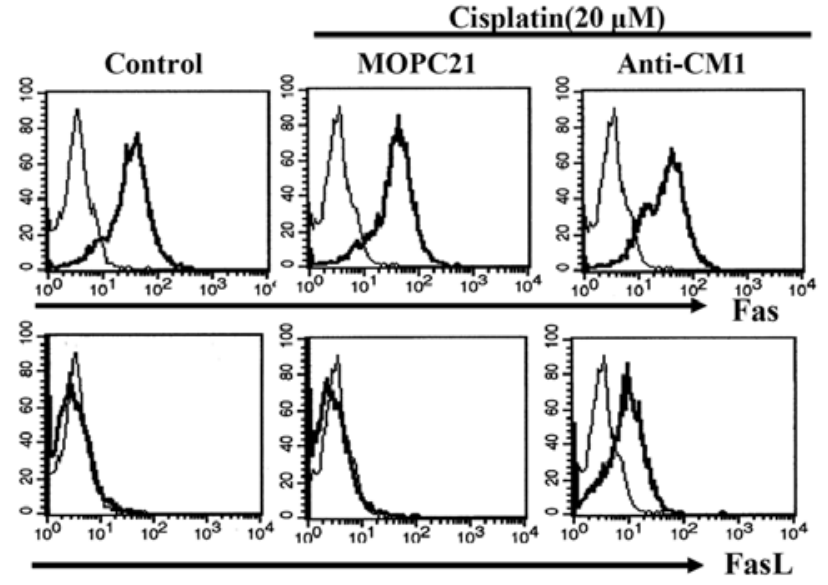

C
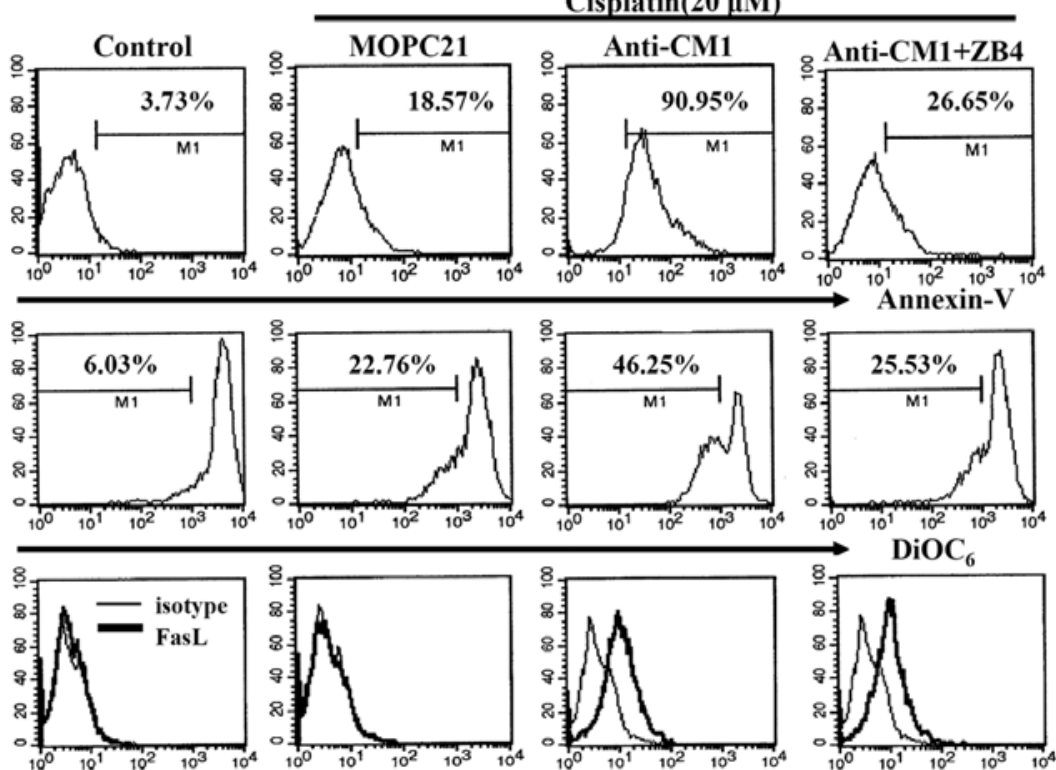

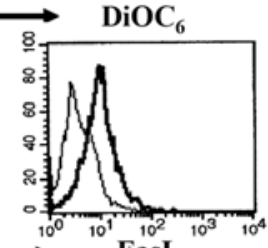

FasL

Figure 4. The effects of inhibition on CM1-mediated apoptosis in cisplatin-exposed HeLa cells. (A) Cisplatin-exposed HeLa cells were pre-incubated with z-IETD fmk ( $20 \mu \mathrm{M}, 2 \mathrm{~h})$ before ligation of CM1 with anti-CM1 antibody. Sixteen hours later, the number of Annexin V-positive cells and mitochondrial membrane potential disruption were obtained as described in Materials and methods. (B) Fas and Fas ligand expression in cisplatin-exposed HeLa cells after CM1 cross-linking. (C) Effects of ZB4 (Fas-blocking antibody) in cisplatin-exposed HeLa cells after CM1 cross-linking. Cisplatin-exposed HeLa cells $\left(1 \times 10^{6}\right.$ cells $\left./ \mathrm{ml}\right)$ were pre-incubated with ZB4 $(0.5 \mathrm{mg} / \mathrm{ml})$ for $1 \mathrm{~h}$. Cells were then washed with PBS and incubated in anti-CM1 or isotype control antibodycoated plates for $40 \mathrm{~min}$. Cells were then cultured in media containing $10 \% \mathrm{FBS}$ at $37^{\circ} \mathrm{C}$ overnight. FITC-conjugated Annexin V staining was performed to assess apoptosis after overnight incubation. The number of $\mathrm{DiOC}_{6}$ represents the percentage of disrupted mitochondrial membrane potentials. Results are representative of three independent experiments.

proteins caused by CM1 ligation in cisplatin-exposed HeLa cells (Fig. 5B and C). These results suggest that the CM1-mediated ROS generation leads to the expression of FasL to generate the apoptosis signal through cleavage of procaspase- 8 .

\section{Discussion}

Cisplatin is conventionally used as chemotherapy for various cancers (9) and is an important first-line drug in advanced cervical cancer. Despite a consistent rate of initial response, cisplatin treatment often results in the development of chemoresistance, which ultimately leads to therapeutic failure (14). Recently, several studies reported that impaired apoptosis involving the caspase cascade and other apoptotic proteins lead to resistance to cisplatin in ovarian and uterine cancers (15-17). Many studies have reported that combination chemotherapy improves outcomes compared to cisplatin monotherapy (15). In recent years, a new paradigm of cancer therapeutics is emerging involving molecularly-targeted therapeutics rather than conventional cytotoxic drugs $(18,19)$. Given the high rate of cisplatin failure in advanced cervical cancer, new anticancer target molecules are urgently needed. Our results suggest that the cross-linking of CM1 with anti-CM1 antibody on cisplatin-exposed HeLa cells represents a novel potential combinational therapy capable of regulating cancer cell death.

It has previously been reported that CM1 cross-linking increases ROS generation and mitochondrial membrane disruption (12). It has also been reported that pretreatment 
A

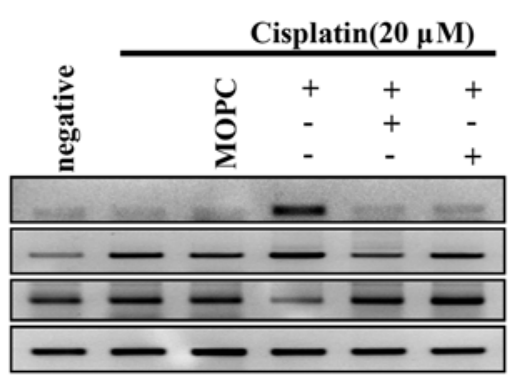

Anti-CM1

z-VAD

NAC

FasL

Bad

Bcl-2

B-actin

B

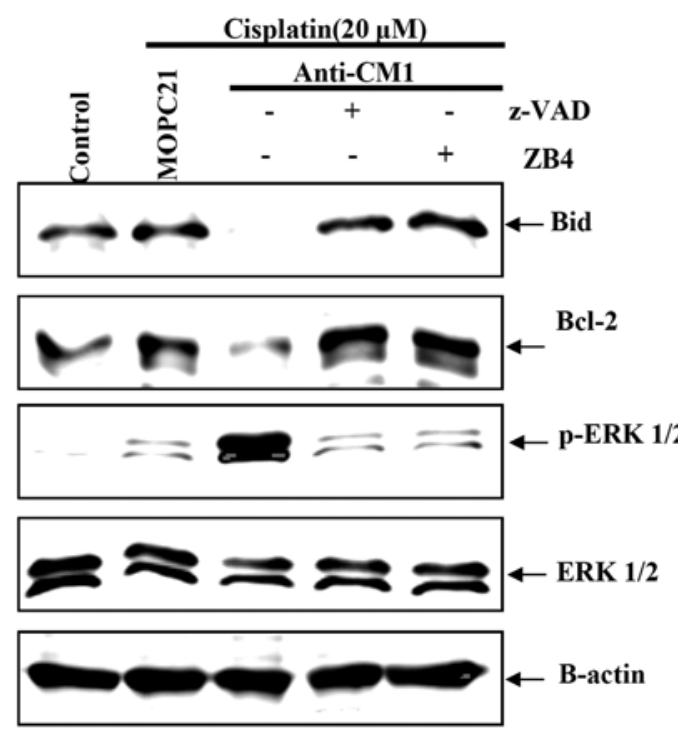

C

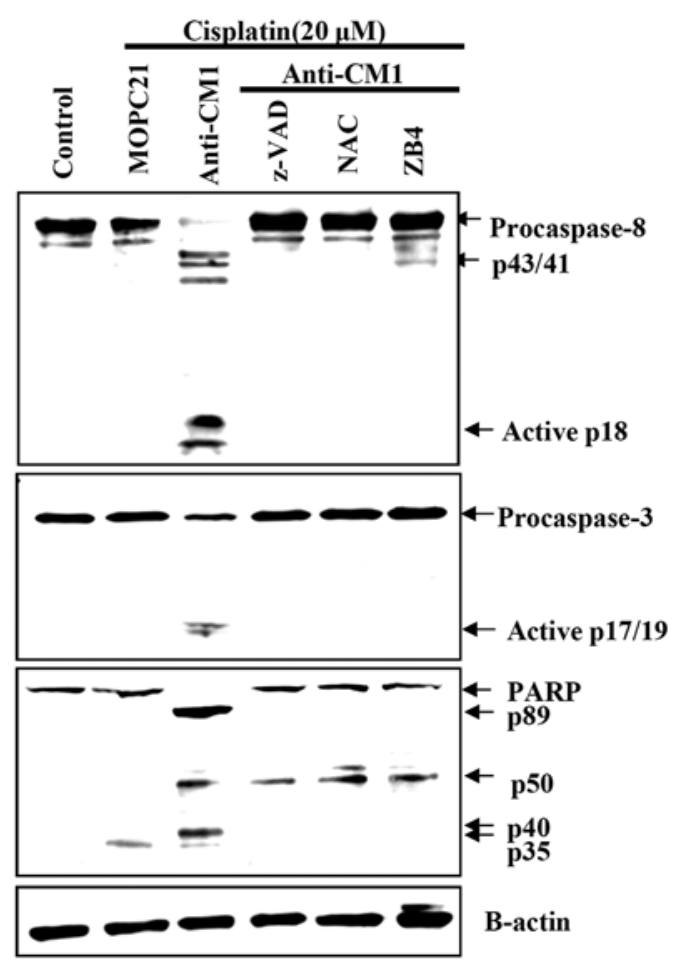

Figure 5. Effect of CM1 ligation on apoptosis-related molecules in cisplatin-exposed HeLa cells. Reverse transcription polymerase chain reaction (RT-PCR) and western blot analysis for apoptosis-associated genes and signaling proteins in cisplatin-exposed HeLa cells after CM1 ligation. (A) Fas ligand and Bcl-2 family mRNA expression in cisplatin-exposed HeLa cells after CM1 cross-linking. Cells were treated as described in Materials and methods, then total RNA was isolated using an RNeasy Mini kit for RT-PCR. (B and C) Western blot analysis was performed to examine the change in apoptosis-related gene products (Bid and Bcl-2), ERK1/2 phosphorylation, caspase activity, and Fas-FasL pathway. Cisplatin-exposed HeLa cells were pre-incubated with or without various inhibitors (Z-VAD, NAC and ZB4) before stimulation of CM1 with anti-CM1 antibody. Results are representative of three independent experiments.

with the pan-caspase inhibitor z-VAD and the ROS quencher, NAC, inhibits CM1-mediated apoptosis in Burkitt's lymphoma cells, EBV-transformed B cells, and lung cancer cells $(12,13)$. Cisplatin-induced cytotoxicity is associated with increased intracellular ROS accumulation and apoptosis mediated by proteolytically activated caspase- 3 and -9 . Several studies have found that ROS generation and therefore apoptosis can be inhibited by upregulating the expression of anti-oxidant proteins and by NAC exposure (20-22). Taken together with this prior research, our results suggest that ligation of CM1 with anti-CM1 antibody generates additional ROS, overcoming the upregulation of anti-oxidants implicated in the rescue mechanism against cancer cell death in cisplatin-exposed HeLa cells.

It has been reported that cisplatin-induced apoptosis is mediated through the Fas/FasL signal transduction pathway in many solid tumors and hematologic malignancies (23-25). Several studies have reported that chemoresistance is correlated with decreased Fas expression (26-28). HeLa cells constitutively express Fas, but not FasL. We found that CM1 cross-linking on cisplatin-exposed HeLa cells significantly increased FasL expression on both protein and RNA levels. In addition, our results show that CM1-mediated apoptosis was blocked after treatment with ZB4, an antagonistic anti-Fas antibody.

The extrinsic apoptosis pathway is triggered by the binding of tumor necrosis factor (TNF)-family death ligands to their appropriate cell surface receptors, followed by caspase- 8 cleavage. The intrinsic apoptosis pathway involves dysregulation of mitochondrial pro- and anti-apoptosis proteins; caspase-8 and Bid, a pro-apoptosis BH3 family member, are then cleaved by the death ligands. Activated caspase- 8 and truncated Bid are correlated with the production of high levels of anti-apoptosis gene products and mitochondrial membrane disruption (29). Similarly, our results showed that CM1 cross-linking disrupts the mitochondrial membrane potential and dramatically increased the release of apoptotic molecules from the mitochondria, such as AIF and EndoG, while decreasing Bcl-2 levels. Pretreatment with Z-VAD-fmk, NAC, or ZB4 completely inhibited apoptosis by blocking the activation of caspase- $8,-3$, and PARP cleavage, as well as normalizing levels of Bcl-2 and Bid proteins, in cisplatin-exposed HeLa cells. These results suggest that CM1-stimulated FasL upregulation generates the apoptosis signal via procaspase- 8 cleavage and mitochondrial membrane disruption in cisplatin-exposed HeLa cells. 
The MAPK signaling cascade plays a pivotal role in regulating cell growth and survival (23). Several studies have reported that cisplatin-induced ERK activation is associated with increased resistance to its cytotoxicity in cancer cells $(30,31)$. Our results showed that CM1 ligation induced the phosphorylation of ERK1/2 in cisplatin-exposed HeLa cells. Treatment of z-VAD-fmk and NAC effectively blocked ERK 1/2 phosphorylation and other changes in apoptosisrelated proteins. Although the correlation between Fas-Fas ligand upregulation and ERK1/2 phosphorylation remains controversial, our results are consistent with a previous report that CM1 ligation induces apoptosis and ERK1/2 phosphorylation in A549 lung cancer cells (13). Some studies have shown that Fas and Fas ligand proteins can be upregulated via p38 MARK/ERK activation $(32,33)$. It has been also reported that ROS induces caspase activity and JNK phosphorylation, as well as participates in ERK1/2 signaling $(34,35)$.

Based on these results, we provide a more detailed understanding of the functions of the CM1 molecule in cervical cancer cells. We propose combination chemotherapy with cisplatin and anti-CM1 antibody as a new therapeutic strategy to overcome chemoresistance in human cervical cancer.

\section{Acknowledgements}

This study was supported by the Korea Research Foundation Grant funded by the Korean Government (313-2008-2-E00023) and a grant from the Korea Healthcare Technology R\&D Project of the Ministry of Health and Welfare Affairs, Republic of Korea (grant no. HI12C0005).

\section{References}

1. Jemal A, Bray F, Center MM, Ferlay J, Ward E and Forman D: Global cancer statistics. CA Cancer J Clin 61: 69-90, 2011.

2. ZurHausen H: Papillomaviruses and cancer: from basic studies to clinical application. Nat Rev Cancer 2: 342-350, 2002.

3. Sankaranarayanan R, Swaminathan R, Brenner H, Chen K, Chia KS, Chen JG, Law SC, Ahn YO, Xiang YB, Yeole BB, Shin HR, Shanta V, Woo ZH, Martin N, Sumitsawan Y, Sriplung H, Barboza AO, Eser S, Nene BM, Suwanrungruang K, Jayalekshmi P, Dikshit R, Wabinga H, Esteban DB, Laudico A, Bhurgri Y, Bah E and Al-Hamdan N: Cancer survival in Africa, Asia, and Central America: a population-based study. Lancet Oncol 11: 165-173, 2010.

4. Al-Mansour Z and Verschraegen C: Locally advanced cervical cancer: what is the standard of care? Curr Opin Oncol 22: 503-512, 2010.

5. Eastman A: Alkylating and platinum-based agents. Curr Opin Oncol 2: 1109-1114, 1990.

6. Brabec V and Kasparkova J: Molecular aspects of resistance to antitumor platinum drugs. Drug Resist Updat 5: 147-161, 2002.

7. Djeu JY and Wei S: Clusterin and chemoresistance. Adv Cancer Res 105: 77-92, 2009.

8. Siddik ZH: Cisplatin: mode of cytotoxic action and molecular basis of resistance. Oncogene 22: 7265-7279, 2003.

9. Eastman A: Mechanisms of resistance to cisplatin. Cancer Treat Res 57: 233-249, 1991.

10. Hur DY, Kim S, Kim YI, Min HY, Kim DJ, Lee DS, Cho D, Hwang YI, Hwang DH, Park SH, Ahn HK, Chang KY, Kim YB and Lee WJ: CM1, a possible novel activation molecule on humanlymphocytes. Immunol Lett 74: 95-102, 2000.

11. Kim D, Hur DY, Kim YS, Lee K, Lee Y, Cho D, Kang JS, Kim YI, Hahm E, Yang Y, Yoon S, Kim S, Lee WB, Park HY, Kim YB, Hwang YI, Chang KY and Lee WJ: CM1 ligation initiates apoptosis in a caspase 8-dependent manner in Ramos cells and in a mitochondria-controlled manner in Raji cells. Hum Immunol 63: 576-587, 2002.
12. Kim YS, Park GB, Choi YM, Kwon OS, Song HK, Kang JS, Kim YI, Lee WJ and Hur DY: Ligation of centrocyte/centroblast marker 1 on Epstein-Barr virus-transformed B lymphocytes induces cell death in a reactive oxygen species-dependent manner. Hum Immunol 67: 795-807, 2006.

13. Lee HK, Park GB, Kim YS, Song H, Broaddus VC and Hur DY: Ligation of CM1 enhances apoptosis of lung cancer cells through different mechanisms in conformity with EGFR mutation. Int $\mathbf{J}$ Oncol 42: 469-477, 2013.

14. Galluzzi L, Senovilla L, Vitale I, Michels J, Martins I, Kepp O, Castedo M and Kroemer G: Molecular mechanisms of cisplatin resistance. Oncogene 31: 1869-1883, 2012.

15. Jin KL, Park JY, Noh EJ, Hoe KL, Lee JH, Kim JH and Nam JH: The effect of combined treatment with cisplatin and histone deacetylase inhibitors on HeLa cells. J Gynecol Oncol 21: 262-268, 2010.

16. Yunos NM, Beale P, Yu JQ and Huq F: Synergism from sequenced combinations of curcumin and epigallocatechin-3-gallate with cisplatin in the killing of human ovarian cancer cells. Anticancer Res 31: 1131-1140, 2011.

17. Zhang $\mathrm{Y}$, Wang $\mathrm{C}$, Wang $\mathrm{H}$, Wang $\mathrm{K}$, Du $\mathrm{Y}$ and Zhang J: Combination of Tetrandrine with cisplatin enhances cytotoxicity through growth suppression and apoptosis in ovarian cancer in vitro and in vivo. Cancer Lett 304: 21-32, 2011.

18. Zagouri F, Sergentanis TN, Chrysikos D, Filipits M and Bartsch R: Molecularly targeted therapies in cervical cancer. A systematic review. Gynecol Oncol 126: 291-303, 2012.

19. Soonthornthum T, Arias-Pulido H, Joste N, Lomo L, Muller C, Rutledge $\mathrm{T}$ and Verschraegen C: Epidermal growth factor receptor as a biomarker for cervical cancer. Ann Oncol 22: 2166-2178, 2011.

20. Chen J, Adikari M, Pallai R, Parekh HK and Simpkins H: Dihydrodiol dehydrogenases regulate the generation of reactive oxygen species and the development of cisplatin resistance in human ovarian carcinoma cells. Cancer Chemother Pharmacol 61: 979-987, 2008.

21. Miyajima A, Nakashima J, Tachibana M, Nakamura K, Hayakawa M and Murai M: N-acetylcysteine modifies cisdichlorodiammineplatinum-induced effects in bladder cancer cells. Jpn J Cancer Res 90: 565-570, 1999.

22. Pak JH, Choi WH, Lee HM, Joo WD, Kim JH, Kim YT, Kim YM and Nam JH: Peroxiredoxin 6 overexpression attenuates cisplatin-induced apoptosis in human ovarian cancer cells. Cancer Invest 29: 21-28, 2011.

23. Brozovic A, Fritz G, Christmann M, Zisowsky J, Jaehde U, Osmak M and Kaina B: Long-term activation of SAPK/JNK, p38 kinase and fas-L expression by cisplatin is attenuated in human carcinoma cells that acquired drug resistance. Int J Cancer 112: 974-985, 2004.

24. Etter AL, Bassi I, Germain S, Delaloye JF, Tschopp J, Sordat B and Dupuis M: The combination of chemotherapy and intraperitoneal MegaFas Ligand improves treatment of ovarian carcinoma. Gynecol Oncol 107: 14-21, 2007.

25. Hougardy BM, van der Zee AG, van den Heuvel FA, Timmer T, de Vries EG and de Jong S: Sensitivity to Fas-mediated apoptosis in high-risk HPV-positive human cervical cancer cells: relationship with Fas, caspase-8, and Bid. Gynecol Oncol 97: 353-364, 2005.

26. Baguley BC: Novel strategies for overcoming multidrug resistance in cancer. BioDrugs 16: 97-103, 2002.

27. Ikuta K, Takemura K, Kihara M, Naito S, Lee E, Shimizu E and Yamauchi A: Defects in apoptotic signal transduction in cisplatin-resistant non-small cell lung cancer cells. Oncol Rep 13: 1229-1234, 2005.

28. Wu W, Wang HD, Guo W, Yang K, Zhao YP, Jiang YG and He P: Up-regulation of Fas reverses cisplatin resistance of human small cell lung cancer cells. J Exp Clin Cancer Res 29: 49, 2010.

29. Elmore S: Apoptosis: a review of programmed cell death. Toxicol Pathol 35: 495-516, 2007.

30. Wang J, Zhou JY and Wu GS: ERK-dependent MKP-1-mediated cisplatin resistance in human ovarian cancer cells. Cancer Res 67: 11933-1141, 2007.

31. Wu ZZ, Sun NK, Chien KY and Chao CC: Silencing of the SNARE protein NAPA sensitizes cancer cells to cisplatin by inducing ERK1/2 signaling, synoviolin ubiquitination and p53 accumulation. Biochem Pharmacol 82: 1630-1640, 2011.

32. Liu WH and Chang LS: Piceatannol induces Fas and FasL up-regulation in human leukemia U937 cells via $\mathrm{Ca}^{2+} / \mathrm{p} 38$ alpha MAPK-mediated activation of c-Jun and ATF-2 pathways. Int J Biochem Cell Biol 42: 1498-1506, 2010. 
33. Duan SG, Cheng L, Li DJ, Zhu J, Xiong Y, Li XW and Wang SG: The role of MAPK-ERK pathway in 67-kDa laminin receptorinduced FasL expression in human cholangiocarcinoma cells. Dig Dis Sci 55: 2844-2852, 2010.

34. Wang M, Zhang L, Han X, Yang J, Qian J, Hong S, Samaniego F, Romaguera J and Yi Q: Atiprimod inhibits the growth of mantle cell lymphoma in vitro and in vivo and induces apoptosis via activating the mitochondrial pathways. Blood 109: 5455-5462, 2007.

35. Filomeni G, Aquilano K, Rotilio G and Ciriolo MR: Reactive oxygen species-dependent c-Jun NH2-terminal kinase/c-Jun signaling cascade mediates neuroblastoma cell death induced by diallyl disulfide. Cancer Res 63: 5940-5949, 2003. 